\title{
Opportunities for the Bioeconomy of Olive Oil Byproducts
}

\author{
J Berbel $^{1 *}$ and A Posadillo \\ ${ }^{1}$ University of Cordoba, Weare, Spain \\ ${ }^{2}$ Seneca Green Catalyst, SL. Córdoba, Spain
}

Received: December 23, 2017; Published: January 04, 2018

*Corresponding author: J Berbel, University of Cordoba, Weare, Spain; Email: berbel@uco.es

\begin{abstract}
Olive oil byproducts are a low cost source for improving fatty by reducing the content of medium chain saturated fatty acids (FA), which have a hypercholesterolemia effect, and increase the fatty acids considered beneficial, such as polyunsaturated FA. This can be done simultaneously reducing the cost of animal feed. This strategy will additionally increase the value of olive oil byproducts that are currently either an environmental problem, or have a low value use (energy or fertilizer). Additionally, research should be done in the analysis of dairy processed products (cheese, yogurts, etc.) in order to test the quality not only from the cardiovascular point of view but also from flavor and other market qualities.
\end{abstract}

Keywords: Olive oil byproducts; Bioeconomy; Milk fat

Abbreviations: FA: Fatty Acids, OL: Olive Leaves; OC: Olive Cake; OS: Olive Stones; OCA: Olive Cake Ash; CSFA: Calcium Soaps of Olive Oil Fatty Acids; OP: Olive Pomace

\section{Introduction}

Bioeconomy is defined as the use of biological sciences for improving adding value to the economic activity (products and services). Bioeconomy refers to the set of economic activities relating to the invention, development, production and use of biological products and processes Oborne [1]. These benefits are expected to improve health outcomes, boost the productivity of agriculture and industrial processes, and enhance environmental sustainability. It is well known that a reduction in dietary saturated fat has generally been thought to improve cardiovascular health. Dietary and policy recommendations frequently focus on reducing saturated fatty acid consumption for improving cardiometabolic health. Milk and dairy foods contain saturated fats, and their consumption often leads to a rise in plasma cholesterol level. Metaanalyses suggest a reduction in risk in the subjects with the highest dairy consumption relative to those with the lowest intake Elwood \& Pickering [2].

There is a growing interest in improving fatty acids prole of milk fat by including appropriate fat sources in dairy ruminant diets. The aim is to reduce the content of medium chain saturated fatty acids (FA), which have a hypercholesterolemia effect, and increase the fatty acids considered beneficial, such as polyunsaturated FA of the $n-3$ series and rumenic acid (cis-9,trans-11 18:2) in milk fat Lock \& Bauman [3]; Martínez Marín, Gómez-Cortés et al. [4]. The source for these polyunsaturated FA acids has been suggested to be oilseeds (sugarbeet, lineseed) and their oils. Furthermore, low quality olive oil has been also used as a source of polyunsaturated FA Castro, Cabezas et al. [5]. Intensive dairy goat, sheep and cow milk production in the Mediterranean basin is based on imported conventional ingredients to be included in concentrates. There is an opportunity for applying the principles of circular economy and bioeconomy to produce milk with lower contain saturated fat by using byproducts of olive oil chain to replace conventional ingredients with the double effect of both reducing feed cost and improving milk quality (and dairy products) relative to human health.

\section{Use of olive oil by-products as animal feedstock}

Olive cultivation is widespread in the Mediterranean regions and the olive tree spread beyond the Mediterranean (e.g. Australia, Argentina, United States of America, etc.). Feeding by-products to livestock is a practice as old as the domestication of animals. Olive byproducts have been used for Mediterranean small ruminants (goat and sheep) and also for dairy cows. Also for non-ruminant olive byproducts have been also been used. By-products derived from the olive trees and olive oil extractions are: Olive leaves (OL) that is to a mixture of leaves and small branches from both, the pruning of olive trees as well as the harvesting and cleaning of olives prior to oil extraction from olives. Olive cake (OC) consists of olive pulp, skin, stone and water although the specific characteristics of OC may differ according type of oil extraction and before and fresh or dry. 
Olive stones (OS) may be a single by-product when they are well separated from pulp either before or after oil extraction. Finally, olive cake ash (OCA) that is the ashes resulting after OC is used as feedstock in biomass energy plant. Additionally, some experiences has been done using low quality oil or calcium soaps of olive oil fatty acids (CSFA). The most frequent used byproduct is the olive pomace (OP) that is one of two major by-products of the olive oil extraction industry, for every $100 \mathrm{~kg}$ of olives, $40 \mathrm{~kg}$ of OP (highly variable depending on technology). Cost reduction is a consequence of the substitution of concentrate feed with a price around $200 \mathrm{EUR} /$ ton for olive byproduct with a cost of $12 \mathrm{EUR} / \mathrm{t}$. The result of this substitution within the recommended limits achieve a $10 \%$ reduction in the cost of goat milk without affecting the yield, Molina-Alcaide, Morales-García et al. [6].

\section{Effects of Olive Oil by-Products as Feedstock in Animal Products}

Nasopoulou and Zabetakis [7] review the use of OP as an alternative dietary lipid source in compounded fish feeds resulting in the formulation of functional fish feeds and aquacultured fish according to the EU legislation (EC 1924/2006). The evidence shows an improvement of meat product with OP improve the cardioprotective properties of the final produce (fish farms) by enriching the fish lipid profile with specific cardioprotective lipid compounds of plant origin Sioriki, Smith et al. [8], Parrillo et al. [9], Nasopoulou \& Zabetakis [7] also quote the use of OP as a feedstock for animal (terrestrial) feed, with some recent research in pigs Martins et al. [10] demonstrating that oleic acid supplementation have complementary effects and influence the nutritional quality of pork and DSF. Regarding ruminants, olive leaves are fibrous with a low digestibility, especially of crude protein, and they promote very poor rumen fermentation. It has also been observed that for lactating animal's olive leaves result in an improvement in milk fat quality compared to diets based on conventional forages MolinaAlcaide \& Yáñez-Ruiz [11].

The knowledge regarding the use of OC is also in line with the evidence of $\mathrm{OL}$, and a significant number of authors have published the evidence. Goat milk shows a significant change in milk composition with increase, with a common result showing that contents of milk fat and milk total solids, as well as their yields, increased in the OC die with a reduction on less saturated fatty acids (FA) and more monounsaturated FA than the control, Castro et al. [5], Gomes et al. [12] and Molina-Alcaide et al. [11]. The mentioned authors agree with the conclusion that the addition of moderate amounts of oil byproducts to the diets of dairy goats has favorable effects on milk fatty acid composition from the point of view of the human consumer, without negative effects on animal performance.

Additionally, research with lactating ewes has been focused in the use of calcium soaps of fatty acids (CSFA) with different original oil (sunflower, soya and olive oil). Results with lactating ewes are also are consistent with the findings for milk goat, with Casals et al. [13], Gargouri et al. [14]; Titi \& Obeidat [15]. Regarding the milk fat contain of cows according the literature review points out that a milk fat reduction is more likely when non-protected fat sources are included in diets based on corn silage but the reduction decreases with other forages Martínez Marín et al. [4], the research in milk cows is less numerous than the evidence for small ruminants (goat and sheep) with OC and other byproducts has not been frequent probably because the high productivity of milk cows and she sensitivity to changes in diet, although some positive experiences have been found with the addition of limited volumes of OL, OC and CSA.

\section{Conclusions and Proposal For Further Research}

This short note has presented the knowledge regarding use of olive oil byproducts, as low cost source for improving fatty acids contain of milk fat by including appropriate fat sources in dairy ruminant diets and therefore to reduce the content of medium chain saturated fatty acids (FA), which have a hypercholesterolemia effect, and increase the fatty acids considered beneficial, such as polyunsaturated FA. There is an opportunity to reduce the cost of dairy feed, and improve health contain of dairy products and additionally increase the value of olive oil byproducts that are currently either an environmental problem, or used as a feedstock for composting or direct field application or in the best of the cases as a fuel for biomass to energy plants. According to the principles of bioeconomy European Commission [16], the use as fertilizer or energy is the less valued alternative with animal feed or human food as prefer end destination of agricultural biomass. The use of these alternative low cost byproducts is done by some farmers without proper control and with ad-hoc formulations and methodology to be converted in a new industry that get higher value for olive byproducts, thus enhancing rural areas and simultaneously improving the quality of dairy products. Additionally, research should be done in the analysis of dairy processed products (cheese, yogurts, etc.) in order to test the quality not only from the cardiovascular point of view but also from flavor and other market qualities.

\section{References}

1. Oborne M (2010) the bioeconomy to 2030: designing a policy agenda. OECD Observer (278): 35-38.

2. Elwood PC, JE Pickering, DI Givens, JE Gallacher (2010) the Consumption of Milk and Dairy Foods and the Incidence of Vascular Disease and Diabetes: An Overview of the Evidence. Lipids 45(10): 925-939.

3. Lock AL, DE Bauman (2004) Modifying milk fat composition of dairy cows to enhance fatty acids beneficial to human health. Lipids 39(12): 1197-1206.

4. Martínez Marín AL, M Pérez Hernández, P Alba, M Luis, D Carrión Pardo, et al. (2013) Fat addition in the diet of dairy ruminants and its effects on productive parameters. Revista Colombiana de Ciencias ecuarias 26(2): 69-78.

5. Castro T, A Cabezas, J De la Fuente, B Isabel, T Manso, et al. (2016) Animal performance and meat characteristics in steers reared in intensive conditions fed with different vegetable oils. Animal 10(3): 520-530.

6. Molina-Alcaide E, EY Morales-García, AI Martín-García, H Ben Salem, A Nefzaoui, et al. (2010) Effects of partial replacement of concentrate with feed blocks on nutrient utilization, microbial $\mathrm{N}$ flow, and milk yield and composition in goats. Journal of Dairy Science 93(5): 2076-2087.

7. Nasopoulou C, I Zabetakis (2013) Agricultural and aquacultural potential of olive pomace a review. Journal of Agricultural Science 5(7): 116. 
8. Sioriki E, TK Smith, CA Demopoulos, I Zabetakis (2016) Structure and cardioprotective activities of polar lipids of olive pomace, olive pomaceenriched fish feed and olive pomace fed gilthead sea bream (Sparus aurata). Food Research International 83: 143-151.

9. Parrillo L, E Coccia, MG Volpe, F Siano, C Pagliarulo, et al. (2017) Olive mill wastewater-enriched diet positively affects growth, oxidative and immune status and intestinal microbiota in the crayfish, Astacus leptodactylus. Aquaculture 473(S): 161-168.

10. Martins JM, A Albuquerque, JA Neves, AB Freitas, R Charneca et al. (2017) Influence of outdo or rearing and oleic acid supplementation on lipid characteristics of muscle and adipose tissues from obese Alentejano pigs. Journal of Animal Physiology and Animal Nutrition.

11. Molina-Alcaide E, Yáñez-Ruiz (2008) Potential use of olive by-products in ruminant feeding: A review. Animal Feed Science and Technology 147(1): $247-264$

12. Gomes LC, CR Alcalde, GT Santos, AC Feihrmann, BSL Molina, et al. (2015) Concentrate with calcium salts of fatty acids increases the concentration of polyunsaturated fatty acids in milk produced by dairy goats. Small Ruminant Research 124(S): 81-88.

13. Casals R, G Caja, MV Pol, X Such, E Albanell, et al. (2006) Response of lactating dairy ewes to various levels of dietary calcium soaps of fatty acids. Animal Feed Science and Technology 131(3): 312-332.

14. Gargouri A, G Caja, R Casals, I Mezghani (2006) Lactational evaluation of effects of calcium soap of fatty acids on dairy ewes. Small Ruminant Research 66(1): 1-10.

15. Titi HH, BS Obeidat (2008) Effects of Ca salt supplementation on milk yield and composition and on lamb growth rate of Awassi ewes. Livestock Science 119(1): 154-160.

16. European Commission (2012) Innovating for Sustainable Growth: A Bioeconomy for Europe. COM (2012) final E Commission Brussels, European Commission.

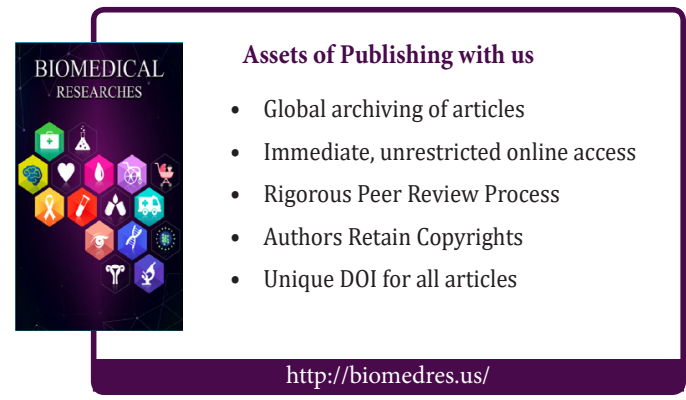

